\title{
Image Segmentation and Asymmetry Analysis of Breast Thermograms for Tumor Detection
}

\author{
Pragati Kapoor \\ Lingaya's University \\ Nachauli, Old Faridabad- \\ Jasana Road, Faridabad- \\ 121002
}

\author{
S. V. A. V. Prasad \\ Lingaya's University \\ Nachauli, Old Faridabad- \\ Jasana Road, Faridabad- \\ 121002
}

\author{
Seema Patni \\ Breast Clinic \\ SLJ Hospital, New Delhi
}

\begin{abstract}
Breast Cancer is the most commonly diagnosed form of cancer in women. Infrared Thermography is a promising technology for breast cancer detection. But analysis of Breast thermograms has often been subjective and has resulted in inconsistency in the diagnosis of breast diseases by thermography. The fast growing tumor has a higher metabolic rate and associated increase in local vascularisation. It will cause the occurrence of some asymmetric heat patterns. Clinical interpretation of a breast thermogram is primarily based on the asymmetry analysis of these heat patterns visually and subjectively. In this paper, a new approach for automatic segmentation of Region of Interest and asymmetry analysis of breast thermograms is implemented. Canny edge detection operator and gradient operator are used to first segment the region of interest. Further asymmetry analysis is performed according to seven extracted features. The abnormality of a breast thermogram is clearly indicated by the features. A GUI is further created in Matlab to make the approach effectual, feasible and for Real Time analysis. 31 thermograms of normal and 11 thermograms of abnormal volunteers were taken.
\end{abstract}

\section{Keywords}

Breast Cancer, Infrared thermography, Breast thermogram, asymmetric heat patterns, automatic segmentation, Region of interest, Canny edge detection operator.

\section{INTRODUCTION}

Breast Cancer together with other breast diseases has become one of the most horrifying experiences in today's women's health. The incidence of breast cancer in India is on the rise and is rapidly becoming the number one cancer in females. India accounts for nearly six percent of deaths due to breast cancer in the world. One out of every 22 women in India is diagnosed with breast cancer [1]

The standard method of mammography to detect tumors has a concern regarding the risk of radiation and patients complain of discomfort due to compression of breasts. In searching for other imaging techniques to complement mammography, infrared thermography can emerge as a potential method to add great practical value in the diagnosing of asymmetric abnormalities for breast using infrared image. Infrared thermography uses a camera with sensitivities in the infrared to provide a picture of the temperature distribution of the human body. It is a non-invasive, non contact, passive, radiation free technique that can also be used in combination with anatomical investigations based on X-rays and often reveals problems when the anatomy is otherwise normal.

Normal tissue that is non-cancerous has a blood supply under the control of the automatic nervous system. Before a cell can become cancerous, the tissues surrounding it start to create new blood vessels. To sustain the rapid growth of these precancerous and cancerous cells a constant supply of nutrients are needed. In order to maintain this supply the "BAD" cells release chemicals in to the surrounding area, which keep existing blood vessels open, awaken dormant ones and create new ones. (This is known as angio-genesis which means new blood vessel growth) The rich vascular beds in the breast provide the conditions necessary for growing tumor's needs. These blood vessels work hard and fast to carry nutrients to the newly formed and extremely hungry cancer cells. All that work feeding these new cancer cells produces additional heat creating "hot spots". Infrared thermography has the ability to detect the temperature or more importantly, actually see the hot spots associated with chemical and blood vessel changes in pre cancerous as well as cancerous breast tissue.

Clinical interpretation of breast thermograms primarily depends on asymmetry analysis. It is nearly impossible to have the tumor symmetrically in both the breasts. The heat patterns occur asymmetrically. The asymmetric heat patterns are usually found visually and analyzed subjectively. Due to limitations of the human visual system including fatigue, boredom and carelessness, these asymmetries might not be easy to detect [2][3][4][5]. In order to eliminate the operator dependency and improve diagnostic accuracy, a new approach for automatic segmentation of Region of Interest and asymmetry analysis of breast thermograms is implemented.

Canny edge detection operator and gradient operator are used to first segment the region of interest [6][7][8]. Further asymmetry analysis is performed according to seven extracted features. The abnormality of a breast thermogram is clearly indicated by the features. The extracted features are classified in three groups - HOS (higher Order Statistics parameters), histogram parameters and centre calculation (Centre of gravity and Geometrical Centre). The HOS include kurtosis, skewness, entropy and Joint Entropy. Based on the values of the HOS, asymmetry ratio is calculated for each volunteer. A GUI is further created in Matlab to make the approach effectual and feasible. 20 thermograms of normal and 6 thermograms of abnormal volunteers were taken. 


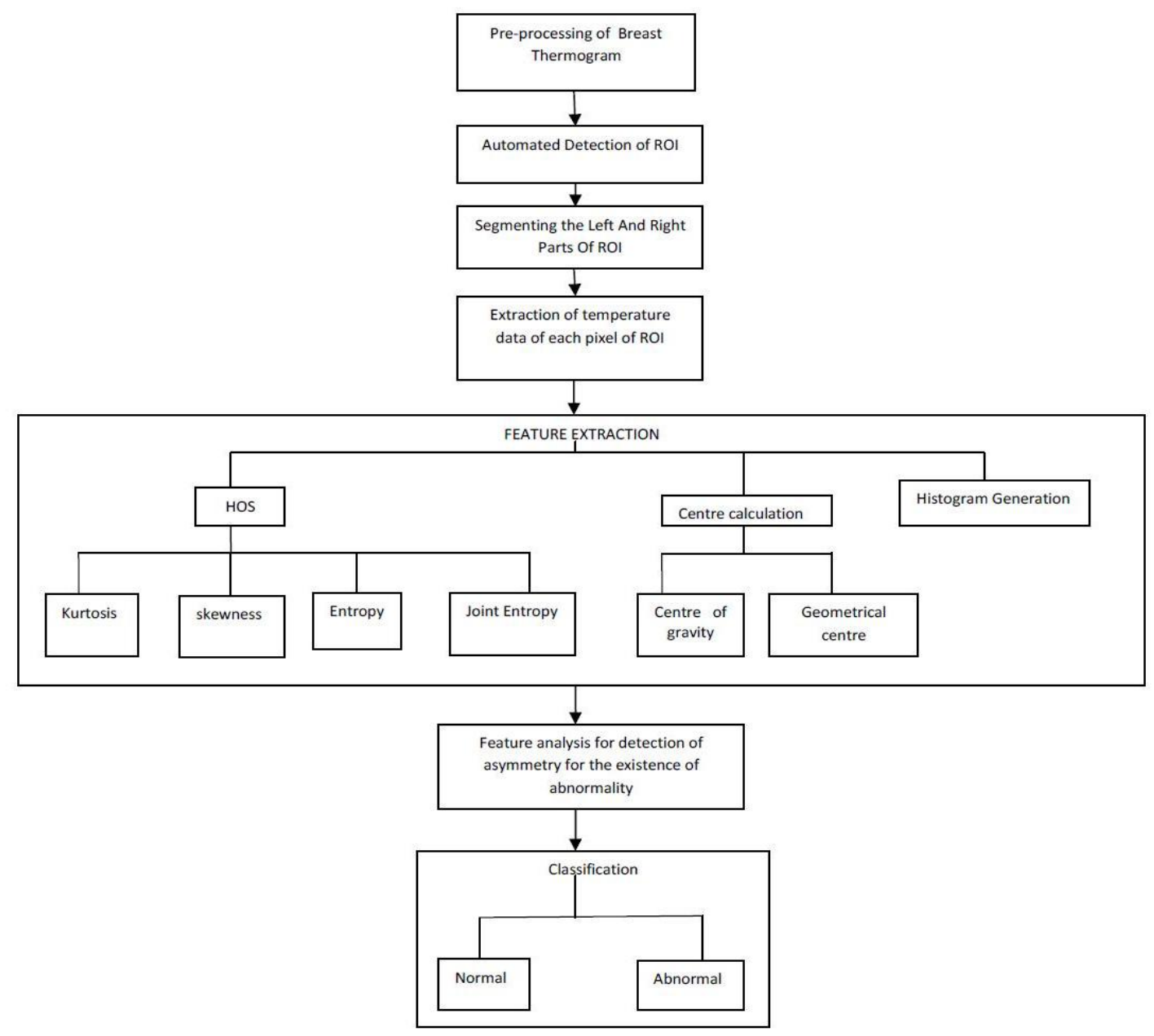

Fig 1: The components of the implemented tumor detection algorithm

\section{AUTOMATED SEGMENTATION OF REGION OF INTEREST (ROI)}

The accuracy of diagnosis depends on how well the segmentation of the region of interest is done [9][10][11]. There are five main steps for segmentation of ROI in breast thermograms for asymmetry analysis. The algorithm implemented is outlined here:

Step I: Pre-processing- Before importing the thermogram image and the pseudo color pallet corresponding to each thermogram into the system they are converted into jpeg format to be processed in Matlab. The First step of preprocessing is Background removal and then resizing the image to remove the undesired body portion. Using the color pallet a matrix is created corresponding to the temperature value of each pixel in the image.

Step II: Canny edge detection operator with threshold value 0 . 4 and sigma 1to extract the boundaries of the breasts. After experimentation it was checked that other values of threshold include noise. Increasing the threshold removes the main curve and decreasing it leads to detection of extra curves.

Step III: Extract the Left and Right boundary curves using Gradient Operator.

Step IV: Extract the two parabolic curves describing the lower boundaries of the breasts. The body boundaries are easy to detect. Difficulties lie in the detection of the lower boundaries of the breasts. We observe that the breast boundaries are generally in parabolic shape. The method uses estimation of all possible curves that can be defined by the parameters - the curvature (a), X and Y coordinates of its lowest peak. This estimation is followed by the selection of the best suitable parameters among them which will describe the actual curve. The process of suitable lower curve detection includes subtraction of average pixel values of successive curves along $\mathrm{Y}$ axis. The two curves whose difference of the average pixel values comes out to be the maximum include the actual breast curves because they yield the maximum difference in their pixel values being different from their nearer region. The same is repeated for the contralateral breast.

Step V: After detection of the lower breast boundaries, the unwanted portions below the breast curves are removed and a middle separation line is created to separate between left and right segments along the point of contact of the two breast curves.

This generates the final segmented image showing two separate breasts from which features can be extracted and asymmetry analysis can be performed. Fig 2 (a) and (b) show the breast thermograms for Volunteer 1(Symptomatic in the left breast with an abnormal mammogram) and Volunteer 2 (Asymptomatic with a normal mammogram) respectively. 


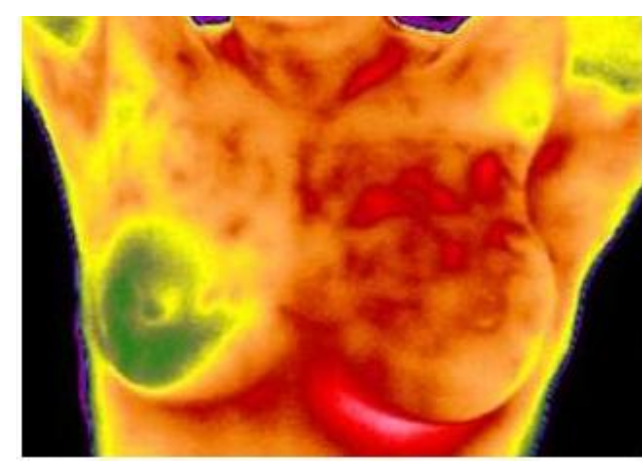

Fig 2: (a) Symptomatic Volunteer 1

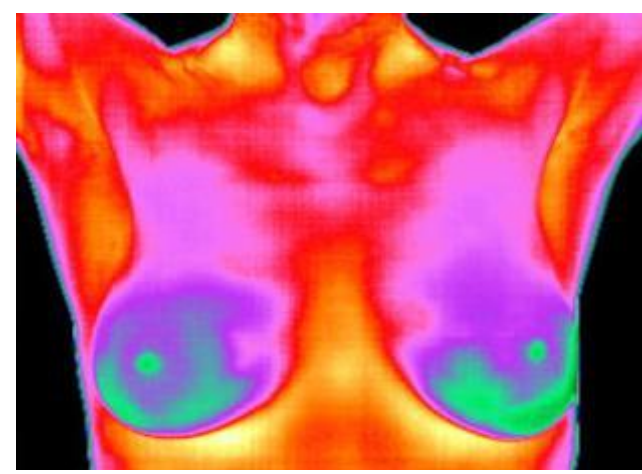

Fig 2: (b) Asymptomatic Volunteer 2

Fig 3 (a) and (b) show the results of automatic segmentation of ROI for Volunteer 1(Symptomatic) and Volunteer 2(Asymptomatic) respectively.

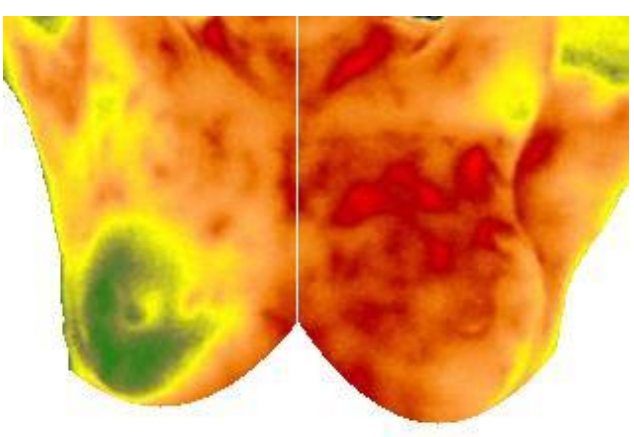

Fig 3: (a) Automated Segmented ROI of Volunteer 1

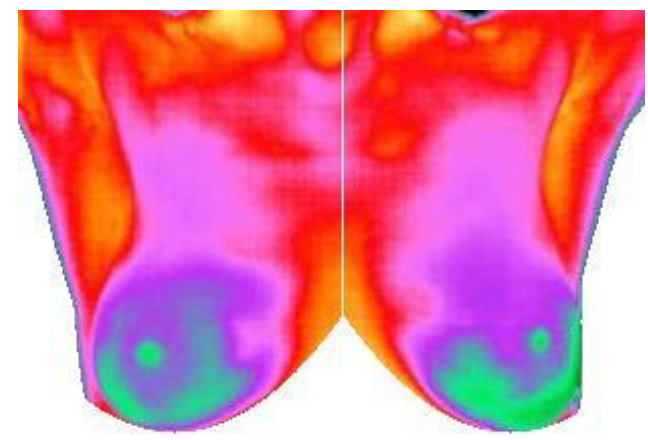

Fig 3: (b) Automated Segmented ROI of Volunteer 2

\section{FEATURE EXTRACTION AND ASYMMETRY ANALYSIS}

Feature Extraction is performed on the segmented thermograms. Extraction and selection of effective features is a necessary step for asymmetry analysis. Therefore, a three way method is adopted to extract features which include higher order Statistical features, Centre calculation and Histogram Generation. Results are shown for Volunteer 1 and Volunteer 2.

\subsection{Higher order Statistical parameters (HOS)}

The HOS based feature extraction includes a comprehensive set of features like Skewness, Kurtosis, Entropy and Joint entropy to capture completely the nature of temperature distribution in the ROI [12][13][14][15].

Skewness: In statistics, skewness is a measure of asymmetry, or more precisely the lack of symmetry. A data set is symmetric if it looks the same to the left and right of the center point (sample mean). Skewness can also be considered a third moment. Its value will vary between contralateral breasts in case of abnormality and will be nearly same for symmetric cases.

Kurtosis: It is a measure of how outlier prone a distribution is. Kurtoses of the contralateral breasts in the ROI help to detect the asymmetries. For symmetrical regions kurtosis value for the contralateral breasts would be the same. Kurtosis can also be considered a fourth moment. Larger kurtosis of a region means more deviation in its symmetry distribution which leads to potential asymmetry.

Skewness $(\gamma 1)$ and kurtosis $(\gamma 2)$ parameters are defined as-

$$
\begin{aligned}
& \gamma_{1}=\frac{E\left[\left(x-E[x]^{3}\right)\right]}{\left(E\left[(x-E[x])^{2}\right]\right)^{3 / 2}} \\
& \gamma_{2}=\frac{E\left[\left(x-E[x]^{4}\right)\right]}{\left(E\left[(x-E[x])^{2}\right]\right)^{2}}-3
\end{aligned}
$$

where $\mathrm{E}[(\mathrm{x}) \mathrm{k}]$ is kth moment of variable $\mathrm{x}$.

Entropy: It measures the uncertainty or the information contained in the segmented images. It measures how much disorder is there in the system. Entropy is hence a useful measure for detecting asymmetries. The more equal the temperature distribution, the more entropy. Therefore the more asymmetric the contralateral breasts are the lower is the Entropy.

Entropy $H(X)=-\sum_{j=1}^{N} p_{j} \log p_{j}$

Joint Entropy: The higher the joint entropy between the left and right breast segments, the more the symmetry and the less possibility of the existence of tumor.

Joint Entropy $H(X, Y)=\sum_{i=1}^{N_{x}} \sum_{j=1}^{N_{y}} p_{i j} \log \left(p_{i j}\right)$

where $p_{i j}$ is the joint probability density. 
Table 1 shows the values of the HOS parameters for Volunteer 1 and Volunteer 2.

Table 1. HOS parameter values

\begin{tabular}{|c|r|c|c|c|}
\hline \multirow{2}{*}{ Feature } & \multicolumn{2}{|c|}{$\begin{array}{c}\text { Volunteer 1 } \\
\text { (Symptomatic) }\end{array}$} & \multicolumn{2}{c|}{$\begin{array}{c}\text { Volunteer 2 } \\
\text { (Asymptomatic) }\end{array}$} \\
\cline { 2 - 5 } & Left & Right & Left & Right \\
\hline Skewness & 1.1910 & 0.3409 & 1.6520 & 1.7955 \\
\hline Kurtosis & 9.7398 & 2.1670 & 8.0113 & 8.7051 \\
\hline Entropy & 15.9552 & 21.1875 & 27.5288 & 28.2562 \\
\hline Joint Entropy & \multicolumn{2}{|c|}{4.3539} & \multicolumn{2}{|c|}{8.1141} \\
\hline
\end{tabular}

Table 1 shows that the values of skewness and kurtosis are quite near in both the breasts for an asymptomatic volunteer, but they are far different in the contralateral breasts of the symptomatic volunteer. Also the entropy values are less in volunteer 1 as compared to volunteer 2 since volunteer 1 has asymmetric heat patterns. The Joint entropy is more for the asymptomatic volunteer 2 with symmetric heat patterns. Fig 4 (a) and (b) illustrate the value of HOS parameters graphically for Volunteer 1 and 2 respectively. $\mathrm{X}$ axis indicates the HOS parameters and $y$ axis indicates their values. For the asymptomatic Volunteer 2, the graphical lines for the left and the right breast nearly coincide.

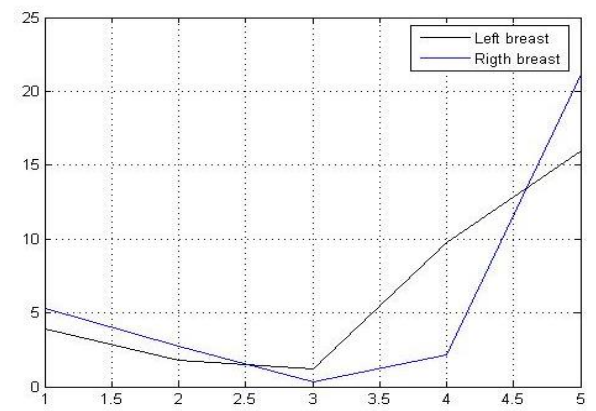

Fig 4: (a) HOS parameters for Volunteer 1

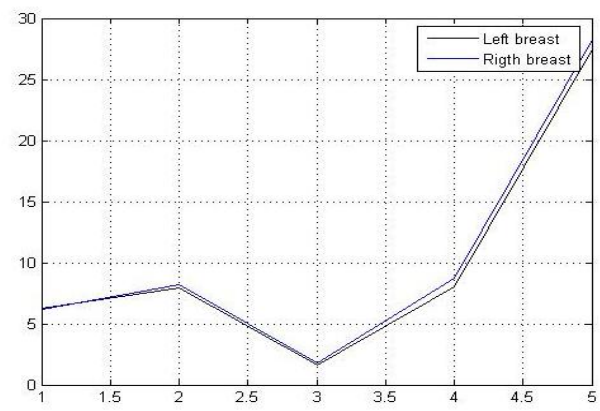

Fig 4: (b) HOS parameters for Volunteer 2

\subsection{Centre Calculation}

We further calculate the centre of gravity (COG) of the breast regions, as well as the distance of the centre of gravity from the geometrical centre of the breast for the left and right segments of the ROI. The centre of gravity is a geometric property of any object. The location of the ROI's centre of gravity will coincide with the geometric centre of the ROI, in case of thermal symmetry. An asymmetrical ROI has a centre of gravity located at some distance from its geometric centre.
Table 2 shows the results of centre calculation.

Table 2. Centre Calculation

\begin{tabular}{|l|c|c|}
\hline Centre calculation & $\begin{array}{c}\text { Volunteer 1 } \\
\text { (Symptomatic) }\end{array}$ & $\begin{array}{c}\text { Volunteer 2 } \\
\text { (Asymptomatic) }\end{array}$ \\
\hline $\begin{array}{l}\text { Difference of COG } \\
\text { and geometrical centre } \\
\text { of left breast }\end{array}$ & 12.324732 & 5.968379 \\
\hline $\begin{array}{l}\text { Difference of COG } \\
\text { and geometrical centre } \\
\text { of right breast }\end{array}$ & 6.771692 & 4.922414 \\
\hline
\end{tabular}

The results obtained in Table 2 show that the difference of $\mathrm{COG}$ and geometrical centre are more in case of thermal asymmetries in the ROI.

\subsection{Histogram Generation}

The histograms generated in Fig 5 (a), (b), (c) and (d) for Volunteer 1 and Volunteer 2 show that the thermal distribution of the ROI for Volunteer 1 is very asymmetric when compared for the contralateral breasts and Volunteer 2 has a fairly symmetrical thermal distribution in both the breasts. The $\mathrm{x}$ axis indicates the temperature values and the $\mathrm{y}$ axis indicates the number of pixels.

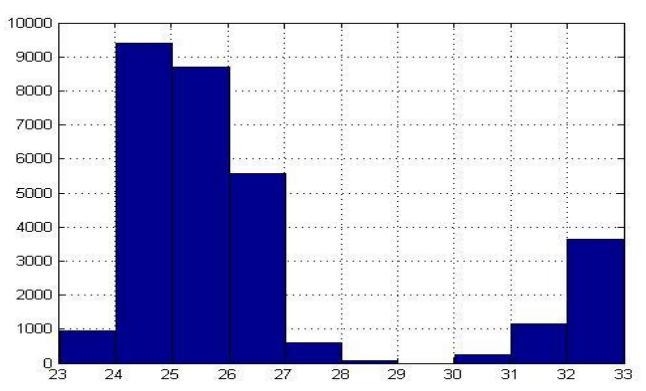

Fig 5: (a) Volunteer 1 Left Breast

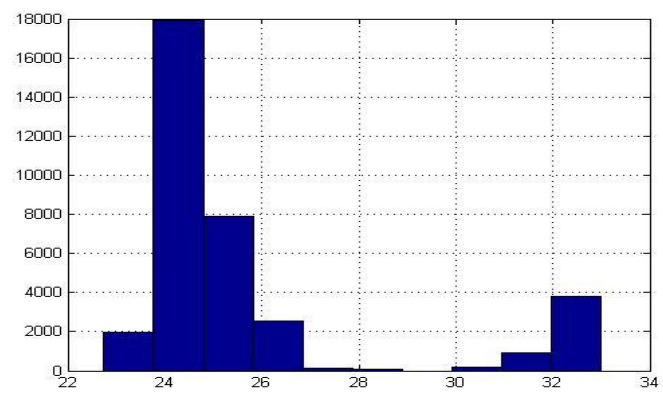

Fig 5: (b) Volunteer 1 Right Breast

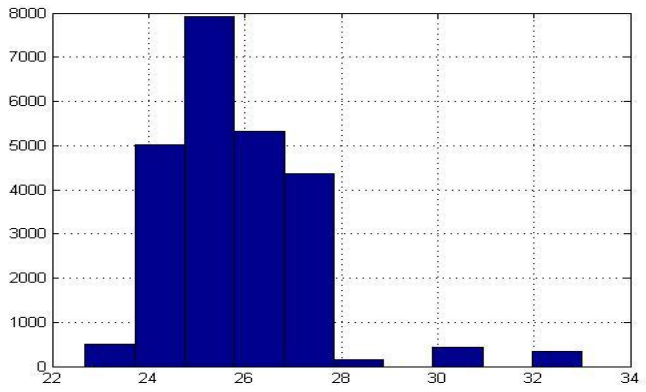

Fig 5: (c) Volunteer 2 Left Breast 


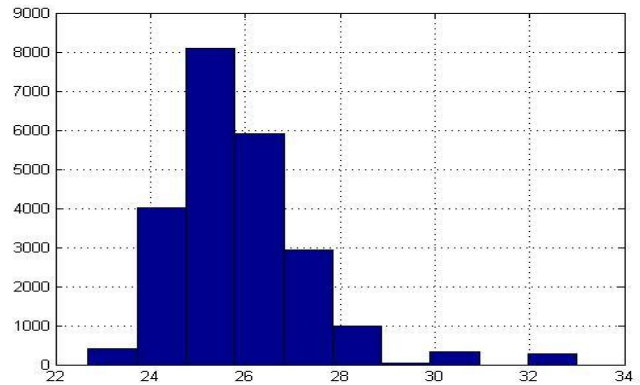

Fig 5: (d) Volunteer 2 Right Breast

\section{GRAPHICAL USER INTERFACE (GUI) FOR REAL TIME ANALYSIS}

A Graphical User Interface is the most common type of user interface seen today. One of the main advantages of creating a standalone GUI is to be able to make it available to a user with no Matlab knowledge [16][17]. Using the Handle Graphics and GUIDE tools of Matlab, a GUI is created with visual elements such as icons, pull down menus, buttons, scroll bars, windows and dialog boxes to simplify user interaction and make the approach effectual and feasible. This human-computer interface would be of great practical value in diagnosing the asymmetric abnormalities for breast using infrared images and will help as a useful second opinion. Fig 6(a) and 6(b) show the GUI created.

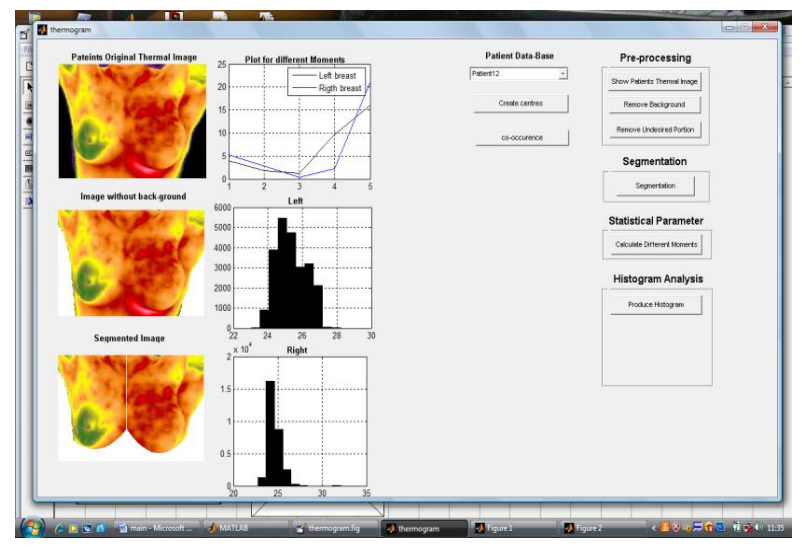

Fig 6 (a): GUI showing details of Volunteer 1

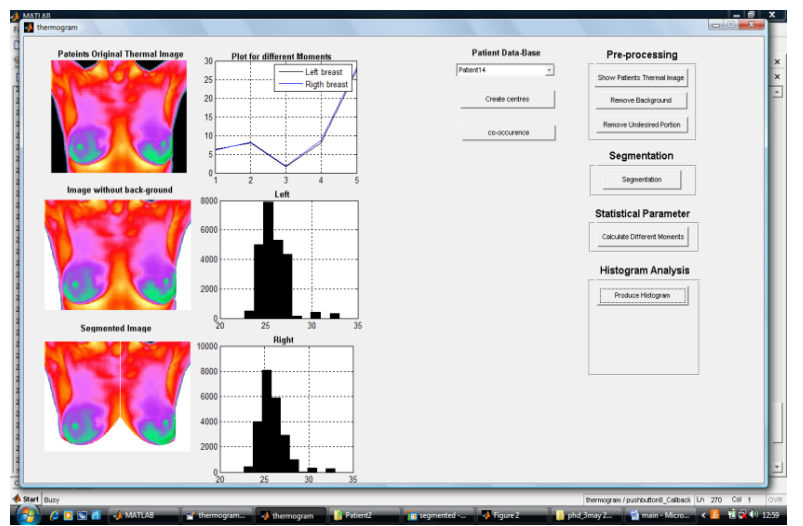

Fig 6 (a): GUI showing details of Volunteer 2
The GUI created allows the users to take full advantage of the powerful multitasking capabilities of modern operating systems. The GUI created has a pull down menu to select any of the patient's breast thermogram. It has push buttons for preprocessing, segmentation, histogram analysis, HOS parameter calculation and centre calculation. It also displays the ROI and histograms and graphical plot of HOS parameters for objective visual analysis. The GUI created is operating System independent and currently supports operating systems such as UNIX, MS Windows, Linux and Mac OS-X. The GUI can be used for Real Time analysis by importing the thermograms captured into Matlab.

\section{CONCLUSION}

Analysis of breast thermography is a labor-intensive task that generally requires careful inspection of small temperature differences and abnormal vascular patterns. In this paper we presented a computational approach to detection of tumor in breast thermograms based on medical infrared imaging. An automated method for identifying the ROI in thermal images is devised using canny edge detector and gradient operator. After an initial stage that involved removing waistlines and shoulders, left, right and lower breast boundaries are identified. Asymmetry analysis is then performed using HOS parameters, centre calculation and histogram generation. Importance of the work is high as the level of asymmetry can predict the degree of future risk and remedial process in advance. The experimental results show that HOS parameters namely skewness, kurtosis, entropy and joint entropy combined with centre calculation and histogram analysis is promising for detection of breast abnormality. Results show that feature extraction is a valuable approach to extract the signatures of asymmetry. This kind of approach will help the diagnostics as a useful second opinion. With a larger database, supervised pattern classification techniques can be used to attain more accurate classification. IR thermography has the benefit of timeliness, no pain, no risk, no contact, non invasive and can be used for mass screening of patients for differential diagnosis of women breast diseases as a powerful adjunct tool to mammography. The GUI's created make the system real time and operating system independent.

\section{ACKNOWLEDGMENTS}

The authors thank 'Tashika Boeki Shokai KK', Japan for providing expertise on high definition thermal imagers. They also want to express their appreciation to E. Y. K. Ng, Adjunct NUH Scientist, office of Biomedical Research, National University Hospital of Singapore for sharing his views and interests on the work.

\section{REFERENCES}

[1] Sherring, Varsha, 2009 Mediating Breast Cancer in India, NCA 94th Annual Convention, San Diego, CA.

[2] Frize, M, Herry, C, Roberge, R, Processing of thermal images to detect Breast Cancer: A comparison, in proc. 2nd Joint IEEE EMBS/BMES Conf., Houston, TX, pp. 1159-1160, 2002.

[3] Mital, M, Scott, E.P., Thermal Detection of Embedded Tumors Using Infrared Imaging, J. Bio-Mechanical. Engineering, 129(1) (2007) 33-39.

[4] Jiang, L. J., Ng, E Y K, Yau, W Y, Jiang, S. Wu X D, A Perspective on Medical IR Imaging, Int. J. Med. Eng. Technol. 29 (6) (2005) 257-267.

[5] Kapoor, Pragati, Prasad, S.V.A.V, et al., 2010 Real Time Intelligent Thermal Analysis approach for early 
diagnosis of breast cancer, International Journal of computer applications, vol.1, no. 5, pp. 22-24.

[6] Hongshan, Yu and Yaonan Wang, 2004 An Improved Canny Edge Detection Algorithm, [J], Computer Engineering and Application, vol 40, pp. 27-29.

[7] Canny, J. A Computational approach to edge detection IEEE Trans, Pattern Anal and Machine Intelligence, vol. 6, 1995 pp.679-698

[8] Qin, Xujia, jiang, Jionghui, Wang, Weihong, Fan, Zhang, Canny operator based level set segmentation algorithm for medical images, 2007, 1st International Conference on Bioinformatics \& Biomedical Engineering [C], ICBBE, 2007, pp. 892-895.

[9] Keyserlingk, J. R., Ahlgren, P. D., Yu, E, Belliveau, N., Yassa, M., Functional Infrared Imaging of the Breast: Historical Perspectives, Current Application and Future Considerations, Biomedical Engineering Handbook, CRC Press, 2006.

[10] Head, Jonathan F, Wang, Fen, Lipari, The Important Role of Infrared Imaging in Breast Cancer, IEEE Engineering in Medicine and Biology, June 2000.

[11] Jones, B.F., A Reappraisal of the Use of Infrared Thermal Image Analysis in Medicine, IEEE Tran. Med Imaging, 17(6) 1998, 1019-1027.
[12] Ng E. Y. K., Ung L.N., et al., 2001 Statistical Analysis of Healthy and Malignant Breast Thermography, Journal of Medical Engineering and Technology, vol. 25, pp. 253263.

[13] Head, J.F., Elliott, R.L., Infrared Imaging: Making Progress in Fulfilling its Medical Promise, IEEE Engineering. Med. Biol. Mag. 21 (2002), 80-85.

[14] G, Schaefer, Zavisek, M., Nakashima, T, Thermography Based Breast Cancer Analysis Using Statistical Features and Fuzzy Classification, Elsevier-Pattern Recognition, 47 (2009) 1133-1137.

[15] Anbar, Michael, Milescu, Lorin, Naumov, Aleksey, Brown, Cheryl, Detection of Cancerous Breasts by Dynamic Area Thermometry, IEEE Engineering in Medicine \& Biology, Sept 2001.

[16] Ng, E.Y.K., Fok, S.C., Peh, Y.C., Ng, F.C. and Sim, L.S.J., 2002 Computerized detection of breast cancer with artificial intelligence and thermograms, International Journal of Medical Engineering \& Technology, 26(4):152-157.

[17] Ng, E Y K, Sudarshan, NM, Computer Simulation in Conjunction With Medical Thermography as an Adjunct Tool For Early Detection of Breast Cancer, BMC Cancer Journal V. 4, 2004 